\title{
Encapsulated bacteriophages in alginate-nanohydroxyapatite hydrogel as a novel delivery system to prevent orthopedic implant-associated

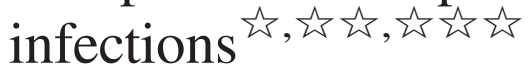

\author{
Joana Alberta Ribeiro Barros, $\mathrm{MsC}^{\mathrm{a}, \mathrm{b}, \mathrm{c}, *}$, Luís Daniel Rodrigues de Melo, $\mathrm{PhD}^{\mathrm{d}}$, \\ Rita Araújo Reis da Silva, $\mathrm{MsC}^{\mathrm{e}}$,f , Maria Pia Ferraz, $\mathrm{PhD}^{\mathrm{g}}$,
} Joana Cecília Valente de Rodrigues Azeredo, $\mathrm{PhD}^{\mathrm{d}}$, Victor Manuel de Carvalho Pinheiro, $\mathrm{PhD}^{\mathrm{h}}$, Bruno Jorge Antunes Colaço, $\mathrm{PhD}^{\mathrm{h}, \mathrm{i}}$, Maria Helena Raposo Fernandes, $\mathrm{PhD}^{\mathrm{e}, \mathrm{f}}$, Pedro de Sousa Gomes, $\mathrm{PhD}^{\mathrm{e}, \mathrm{f}}$, Fernando Jorge Monteiro, $\mathrm{PhD}^{\mathrm{a}, \mathrm{b}, \mathrm{c}}$

${ }^{a} I 3 S$ - Instituto de Investigação e Inovação em Saúde, Universidade do Porto, Porto, Portugal

${ }^{\mathrm{b}}$ INEB - Instituto de Engenharia Biomédica, Universidade do Porto, Porto, Portugal

${ }^{\mathrm{c}}$ FEUP - Faculdade de Engenharia, Universidade do Porto, Porto, Portugal

${ }^{\mathrm{d}}$ Laboratório de Investigação em Biofilmes Rosário Oliveira, Center of Biological Engineering, University of Minho, Braga, Portugal

${ }^{\mathrm{e}}$ Laboratory for Bone Metabolism and Regeneration - Faculty of Dental Medicine, University of Porto, Porto, Portugal

${ }^{\mathrm{f}}$ REQUIMTE/LAQV, University of Porto, Porto, Portugal

${ }^{\mathrm{g}}$ FP-ENAS/CEBIMED - University Fernando Pessoa Energy, Environment and Health Research Unit/Biomedical Research Center, Porto, Portugal

${ }^{\mathrm{h}}$ Department of Animal Sciences, ECAV, University of Trás-os-Montes e Alto Douro, Vila Real, Portugal

${ }^{\mathrm{i}}$ Center for the Research and Technology of Agro-Environmental and Biological Sciences, University of Trás-os-Montes e Alto Douro, Vila Real, Portugal

Received 1 April 2019; revised 26 November 2019; accepted 9 December 2019

\begin{abstract}
An innovative delivery system based on bacteriophages-loaded alginate-nanohydroxyapatite hydrogel was developed as a multifunctional approach for local tissue regeneration and infection prevention and control. Bacteriophages were efficiently encapsulated, without jeopardizing phage viability and functionality, nor affecting hydrogel morphology and chemical composition. Bacteriophage delivery occurred by swelling-disintegration-degradation process of the alginate structure and was influenced by environmental $\mathrm{pH}$. Good tissue response was observed following the implantation of bacteriophages-loaded hydrogels, sustaining their biosafety profile. Bacteriophagesloaded hydrogels did not affect osteoblastic cells' proliferation and morphology. A strong osteogenic and mineralization response was promoted through the implantation of hydrogels system with nanohydroxyapatite. Lastly, bacteriophages-loaded hydrogel showed excellent antimicrobial activity inhibiting the attachment and colonization of multidrug-resistant $E$. faecalis surrounding and within femoral tissues. This new local delivery approach could be a promising approach to prevent and control bacterial contamination during implantation and bone integration.
\end{abstract}

(C) 2020 Elsevier Inc. All rights reserved.

Key words: Bacteriophages; Alginate-nanohydroxyapatite; Biocompatibility; Antimicrobial activity; Osteogenic differentiation

is Conflicts of interest statement: The authors have no conflict of interest to declare.

is Funding: This work was supported by FEDER - Fundo Europeu de Desenvolvimento Regional funds through the COMPETE 2020 - Operational Programme for Competitiveness and Internationalisation (POCI), Portugal 2020, by Portuguese funds through FCT/MCTES in the framework of the project "institute for Research and Innovation in Health Sciences (POCI-01-0145-FEDER-007274), by Project Biotherapies (NORTE-01-0145-FEDER-000012) and by Joana Barros' PhD grant (SFRH/BD/102148/2014). This work was also supported by Portuguese funds through FCT/MCTES (UID/QUI/50006/2019).

论坛话 Acknowledgments: The authors acknowledge the technical support of Rui Fernandes and Rossana Correia, from i3S Scientific Platform - Histology and Electron Microscopy (HEMS), national infrastructure PPBI - Portuguese Platform of Bioimaging (PPBI-POCI-01-0145-FEDER-022122), in the histological and TEM analysis. The support of Dr. Liliana Grenho (FMDUP) in "in vivo" and "in vitro" biocompatibility analysis and "ex vivo" analysis is also deeply appreciated.

*Corresponding author at: Instituto de Investigação e Inovação em Saúde, Rua Alfredo Allen 208, 4200-135 Porto, Portugal.

E-mail address: joana.barros@ineb.up.pt. (J.A.R. Barros). 
Graft implantation is the most used technique for bone loss repair and augmentation, in orthopedic surgical approaches. ${ }^{1}$ However, the implantation procedure is highly susceptible to bacterial infection, due to either locally recruited microorganisms or hematogenous spread of existing pathogens. ${ }^{2,3}$ Current curative approaches, as surgical debridement and prolonged systemic antibiotic therapy often translate into high risk of lifelong functional impairment and morbidity and contribute to increasing economic burden in healthcare systems. ${ }^{3-5}$ Local strategies as vehicles for antimicrobial agents delivery has emerged as an adjunct in the prevention and treatment of bone graft-related infections. ${ }^{3,}$ 6-8 Local delivery systems show advantages over systemic approaches, such as drug delivery at or close to the infection site, increased efficacy and bioavailability of the antimicrobial agent, dose reduction and decreased systemic toxicity risks. ${ }^{8}$ However, some limitations are associated to these systems, namely delivery of sub-therapeutic antimicrobial levels, favoring bacterial resistance development; limited antimicrobial diffusion into the peri-implant tissues; dose-dependent antimicrobial activity, and potential cytotoxicity of the antimicrobial agents. ${ }^{8,9}$ Indiscriminate use of antibiotics has vastly contributed to the emergence of antibiotic-resistant bacteria, increasing its pathogenicity leading to defaulting treatment of infections caused by them. ${ }^{10,11}$ To overcome such limitations, new therapeutics are urgently needed.

Bacteriophages (phages) have emerged as an alternative approach to current antimicrobial chemotherapy due to their capacity to infect and kill specific bacterial strains, without modifying the established commensal microbiome. ${ }^{9}, 10,12-15$ Phages are virulent viruses that recognize specific receptors in bacteria, injecting their genetic material and using the host biochemical machinery to replicate new phages' particles and enzymes, responsible for subsequent bacterial lysis. Furthermore, newly formed phages particles are released spreading and infecting other target bacteria nearby. ${ }^{10,16}$ Phages' propagation and persistence always depend on its host bacterial pathogen presence, contributing to regulate phages number in direct relation with pathogen level facilitating diffusion into surrounding areas in a gradient directly dependent on pathogen availability. ${ }^{9}, 10,12$ Likewise, phages are not pathogenic for eukaryotic cells. Despite being able to interact with phagocytic cells and be adsorbed through mammalian cells' surface receptors, being subsequently phagocytosed and degraded, they are not threatening. ${ }^{10,12,17}$

Several studies have explored the potential of systemic phages administration for the treatment of implant-related infections. ${ }^{13-15}$, 18-21 Nonetheless, experimental studies using phages-loaded biomaterials for local delivery approaches are scarce, $, 22,23$ focusing on the phages release and antimicrobial activity, without simultaneously exploring the potential combination with a regeneration inductive biomaterial. Therefore, this work aims to develop a multi-functional regenerative biomaterial for local phage delivery, based on an alginate-nanohydroxyapatite (Alg-nanoHA) hydrogel system. To the best of our knowledge, the present work is novel in addressing phage-loaded biomaterials as a novel local delivery system, simultaneously promoting bone tissue regeneration, and preventing local tissue infections. The efficacy and safety of this new system were evaluated in vitro, ex vivo and in vivo.

\section{Methods}

Hydrogel system preparation and physico-chemical characterization

Initially, phages-free hydrogels (Alg and Alg-nanoHA) were prepared, that were used as controls. Briefly, a $2 \%(\mathrm{w} / \mathrm{v})$ alginate solution (Alg) was obtained by dissolving sodium alginate powder in distilled water, at room temperature. This solution was mixed with nanoHA powder (nanoXIM.HAp202, FLUIDINOVA®) at $30 \mathrm{wt} \%$, during $1 \mathrm{~h}$ at $60 \mathrm{rpm}$. After homogeneity, mixture was dropped into a $250 \mathrm{mM}$ calcium chloride $\left(\mathrm{CaCl}_{2}\right)$ solution and was allowed to harden for $30 \mathrm{~min}$. Afterward, hydrogels were rinsed twice with PBS and kept at $4{ }^{\circ} \mathrm{C}$. To disclose the relevance of nanoHA within the hydrogel system, Alg hydrogels were similarly prepared, but in the absence of nanoHA.

Following, E. faecalis phages vB_EfaS_LM99 (LM99), previously described by Barros et al., ${ }^{24}$ were encapsulated into hydrogels. Phages LM99 solution $\left(10^{8} \mathrm{PFU} / \mathrm{mL}\right)$ was suspended in the Alg and Alg-nanoHA solutions. Then, Alg + phage LM99 and Alg-nanoHA + phage LM99 mixtures were dropped into a crosslinking solution to obtain hydrogels, under the same conditions as above mentioned.

Phages-loaded hydrogels structure was observed by scanning electron microscopy (SEM). Samples were coated with a thin goldpalladium layer (SPI-Module) in an argon atmosphere and examined using an FEI Quanta 400FEG/ESEM microscope. Phages-free Alg and Alg-nanoHA hydrogels were used as controls.

Phages-loaded hydrogels were further observed by transmission electron microscopy (TEM). Briefly, samples were fixed in $2.5 \%$ glutaraldehyde and $2 \%$ paraformaldehyde in cacodylate Buffer 0.1M (pH7.4), dehydrated and embedded in Epon resin. Ultrathin sections were prepared and stained with uranyl acetate and lead citrate for $15 \mathrm{~min}$ each and examined under a JEOL/JEM 1400 microscopy (TEM). Images were digitally recorded using a CCD digital camera Orious 1100W. Phages-free Alg and AlgnanoHA hydrogels were used as controls.

Chemical characterization of phages-loaded hydrogels was performed using attenuated total reflectance - Fourier transformed infrared spectroscopy (ATR - FTIR), using a Perkin Elmer 2000 FTIR spectrometer. The samples were analyzed at a spectral resolution of $2 \mathrm{~cm}^{-1}$ and 100 scans were accumulated per sample. Phages-free Alg and Alg-nanoHA hydrogels were used as controls.

\section{Functional characterization}

Encapsulation efficiency of the phages and hydrogel system shelf-life assessment

To evaluate the efficiency of phages' encapsulation, phagesloaded hydrogels were dissolved in a microsphere-broken solution (MBS) containing $50 \mathrm{mM}$ sodium citrate, $0.2 \mathrm{M}$ sodium bicarbonate, and $50 \mathrm{mM}$ Tris- $\mathrm{HCl}, \mathrm{pH} 7.5$, for $3 \mathrm{~h}$ under shaking at room temperature. Stability of phage LM99 in MBS was previously tested and validated. Phages encapsulation was quantified by the double-agar-layer plating method ${ }^{25}$ and expressed in PFU/mL. Encapsulation efficiency (EE) was calculated as described elsewhere. ${ }^{26}$ The encapsulated phages shelf-life was tested in Alg and Alg-nanoHA solutions, before 
A)

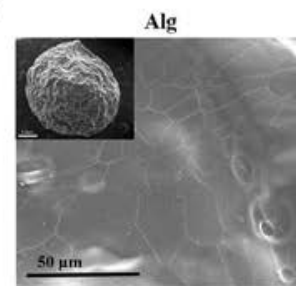

Alg-nanoHA

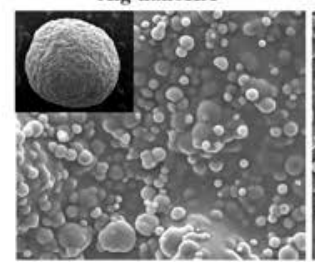

Alg + phage LM99

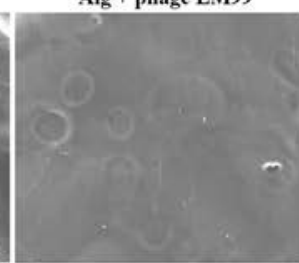

Alg-nanoHA + phage LM99

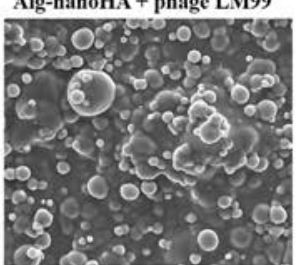

C)

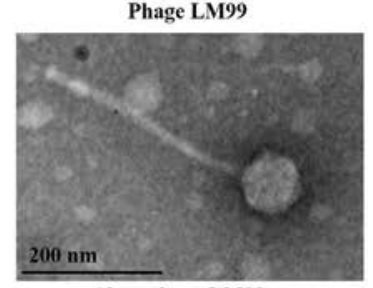

B)

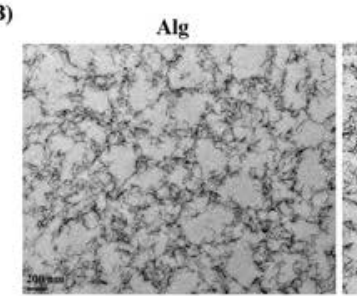

Alg-nanoHA

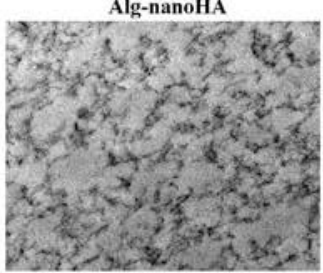

Alg + phage LM99

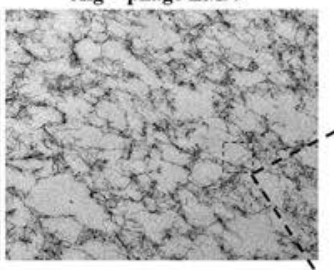

Alg-nanoHA + phage LM99

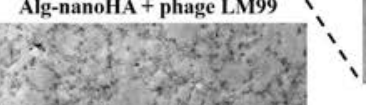

Alg + phage LM99

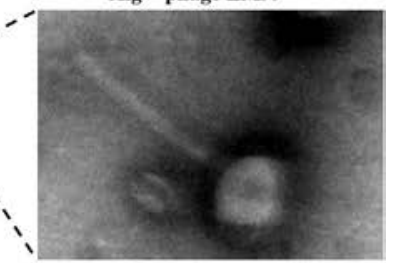

Alg-nanoHA + phage LM99

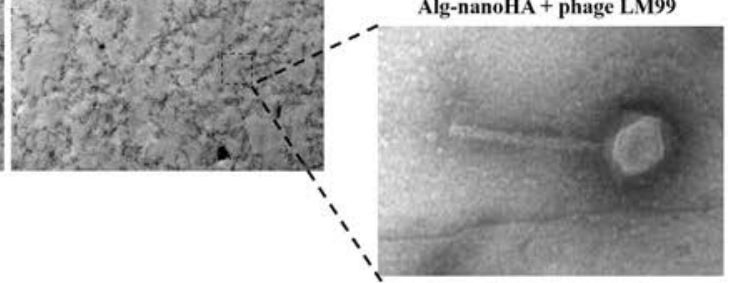

Figure 1. (A) SEM micrographs of nanoHA dispersed through Alg matrix. Scale bar 1mm and $50 \mu \mathrm{m}$. (B) TEM micrographs of Alg network and of (C) phages structures-free hydrogels and encapsulated into hydrogels. Scale bar $200 \mathrm{~nm}$.

crosslinking, and on hydrogels, after crosslinking. From solutions stored at $4^{\circ} \mathrm{C}$, and after the dissolution of hydrogels in MBS, the phages titer was quantified and expressed in PFU/ $\mathrm{mL}$ plotted against time. Three independent experiments were performed in triplicate.

Effect of $\mathrm{pH}$ on the structural behavior of hydrogels and influence on phages release

The effect of $\mathrm{pH}$ on the swelling behavior of the hydrogels and associated phages release was determined by the quantification of the swelling ratio and phages titer after $24 \mathrm{~h}$. The hydrogels swelling behavior was evaluated and calculated by measuring the changes in sample weight as a function of sample incubation in $\mathrm{PBS}$ at $37^{\circ} \mathrm{C}$, for $24 \mathrm{~h}$, as described in the literature. ${ }^{27} \mathrm{Alg}$ and $\mathrm{Alg}$ nanoHA hydrogels phages-free were used as controls. The phages released from hydrogels were quantified by the double-agar-layer plating method and expressed in PFU/mL. The results were taken as the mean values of eight measurements. The experiments were performed in triplicate.

\section{Profile of phages LM99 release}

The profile of phages release from the hydrogels was assayed under dynamic conditions in $\mathrm{PBS}$ at $150 \mathrm{rpm}$ and $37^{\circ} \mathrm{C}$. At predetermined time points, cumulative released phages were quantified using the double-agar-layer plating method. The cumulative amount of released phages was plotted against time. Three independent experiments were performed in triplicate.

\section{Biological characterization}

Inflammatory response - in vivo subcutaneous tissue implantation

The experiment was approved by the Local Ethics Committee and the national regulatory entity - Direção Geral de
Alimentação e Veterinária (DGAV), observing the technical standards of protection for experimental animals, according to both policies and principles of laboratory animal care and with the European Union guidelines (European Directive 2010/63/EU and National Decree-Law 113/2013). Housing, anesthesia, and post-operative care were performed according to Silva et al. ${ }^{7}$ Sixteen New Zealand white rabbits, 18 weeks old and weighing between 3700-3900 g, were used for the subcutaneous implantation of the phages-loaded hydrogels. Briefly, a cutaneous incision (about $3 \mathrm{~cm}$ long) was performed in the dorsal region for the implantation of hydrogels following blunt subcutaneous dissection. Hydrogels were randomly implanted and after hemostatic control, the surgical wound was terminated with Polyglactin 910-4/0 restorable suture, with single stitches. In each animal, three hydrogels were implanted. Two and six weeks after implantation, the animals were euthanized with an anesthetic overdose. The subcutaneous tissue with hydrogel implants was collected by debridement and fixed for conventional histological preparation. Tissue samples were included in paraffin and, following section, stained with hematoxylin and eosin (H\&E). Histological analyses were performed following image acquisition with an Olympus BX-51/22 dotSlide digital virtual microscope. Phages-free Alg and Alg-nanoHA hydrogels were used as controls.

\section{Osteogenic response}

In vitro cytocompatibility assessment with human osteoblastic cells

In vitro cytocompatibility of phages-loaded hydrogels was studied using human osteoblast-like cells (MG63), following cell culture characterization in the presence of hydrogels' eluents. These were collected under dynamic conditions, following 
A)

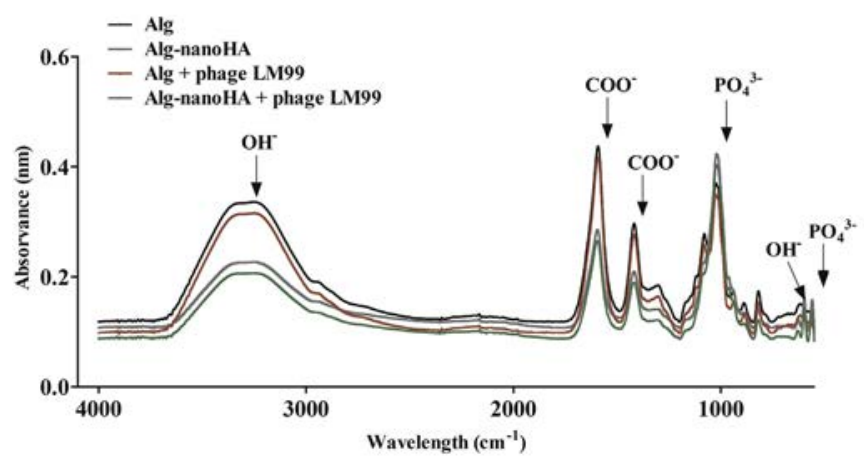

C)

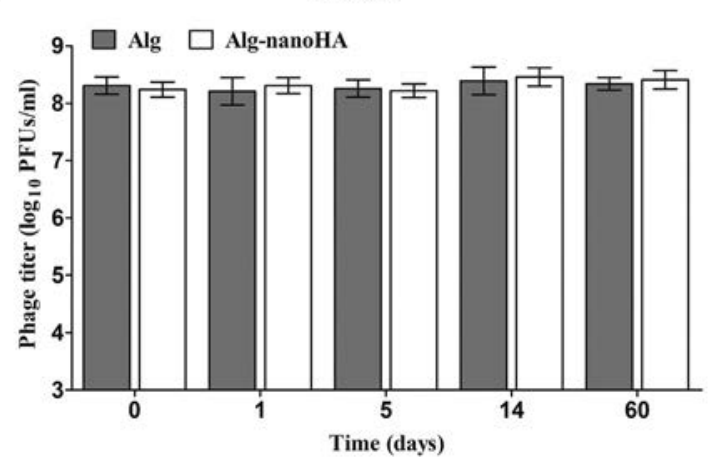

B)

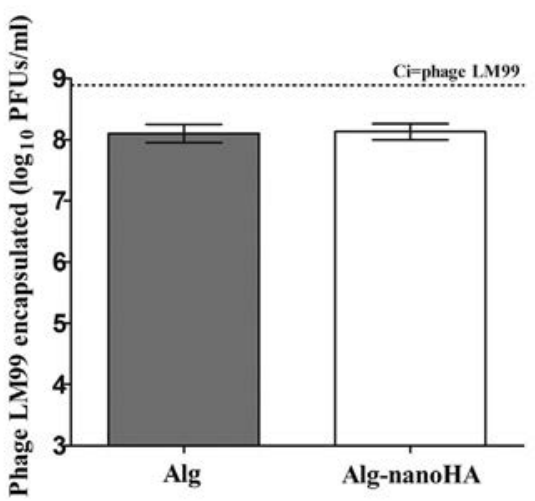

D)

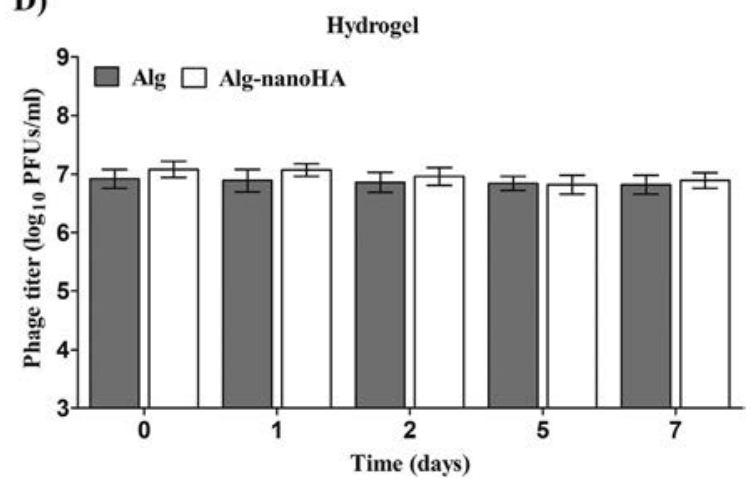

Figure 2. (A) ATR-FTIR spectra of the hydrogels. (B) Encapsulation efficiency of phages on hydrogels. (C, D) Phages titer into solutions and hydrogels, respectively. Quantitative data are expressed in mean \pm SD.

hydrogels incubation in $\alpha$-minimum essential medium $(\alpha-$ MEM), for $24 \mathrm{~h}$, at $37^{\circ} \mathrm{C}$, and at $150 \mathrm{rpm}$. Phages-free $\mathrm{Alg}$ and Alg-nanoHA hydrogels were used as controls. Culture medium without hydrogels was used as a reference control.

Cell cultures were grown in $\alpha$-MEM supplemented with $10 \%$ fetal bovine serum (FBS), $2.5 \mu \mathrm{g} / \mathrm{mL}$ amphotericin B, $100 \mathrm{IU} /$ $\mathrm{mL}$ penicillin and $100 \mu \mathrm{g} / \mathrm{mL}$ streptomycin at $37^{\circ} \mathrm{C}$, in a humidified atmosphere of $5 \% \mathrm{CO}_{2}$ in the air. For sub-culture, adherent cells were enzymatically released with $0.05 \%$ trypsin in $0.25 \%$ EDTA, for $5 \mathrm{~min}$ at $37^{\circ} \mathrm{C}$. and seeded at $10^{4}$ cells $/ \mathrm{cm}^{2}$. After $24 \mathrm{~h}$, cells were exposed to hydrogels' eluents for up to seven days. Biologic response was evaluated in terms of cell morphology, metabolic activity and alkaline phosphatase (ALP) activity.

At each time-point, cultures' metabolic activity was evaluated using the MTT assay, based on the reduction of 3-(4,5dimethylthiazol-2-yl)-2,5-diphenyltetrazolium bromide to a purple formazan product by viable cells, as previously described. ${ }^{28}$

ALP activity was determined as described in literature. ${ }^{28}$ ALP results were normalized to total protein content and expressed as nanomoles of p-nitrophenol produced per microgram of protein. Total protein content was quantified using the DC protein assay Kit (BioRad).

Cell cytoskeleton filamentous actin (F-actin) organization and the osteoblastic cells morphology were assessed by immunofluorescence imaging. Cells were washed with PBS and fixed with
$3.7 \%$ paraformaldehyde for $15 \mathrm{~min}$. Following cells' permeabilization with $0.1 \%(\mathrm{v} / \mathrm{v})$ Triton $\mathrm{X}-100$, nonspecific binding sites were blocked with $1 \%$ bovine serum albumin for $30 \mathrm{~min}$. Factin was stained with Alexa-Fluor 488 phalloidin-conjugated antibody (1:100, $30 \mathrm{~min})$, and nucleus counterstaining with DAPI $(1 \mu \mathrm{g} / \mathrm{mL}, 10 \mathrm{~min})$. Images of fluorescent-labeled cells were obtained with a Celena $\mathrm{S}$ digital imaging system (Logos Biosystems). Three independent experiments were performed in quadruplicates.

\section{Bone formation ex vivo functional assessment}

Osteogenic tissue response was assayed via ex vivo within an embryonic chick femoral segmental defect model. This experiment was carried out in accordance with guidelines and regulations laid down in the Animals (Scientific procedures) act 1986 and with Home office approval UK (Project license PPL30/2762). Femurs were dissected from 11 day-old chick embryos (Gallus domesticus) and cut at middle diaphysis for the establishment of a segmental defect. Then, phages-loaded hydrogels were implanted within the produced defects and were carefully placed into Netwell ${ }^{\mathrm{TM}}$ Insert in six-well-plates. Femurs, with implanted hydrogels, were kept for 11 days in minimum essential medium ( $\alpha$-MEM), containing ascorbic acid $(50 \mu \mathrm{g} / \mathrm{mL})$, penicillin $(100 \mathrm{U} / \mathrm{mL}) /$ streptomycin $(100 \mu \mathrm{g} / \mathrm{mL})$ and $2.5 \mu \mathrm{g} / \mathrm{mL}$ amphotericin $\mathrm{B}$, at the liquid/gas interface, in a humidified atmosphere of $5 \% \mathrm{CO}_{2}$ in air, and $37^{\circ} \mathrm{C}$. Culture 
A1)

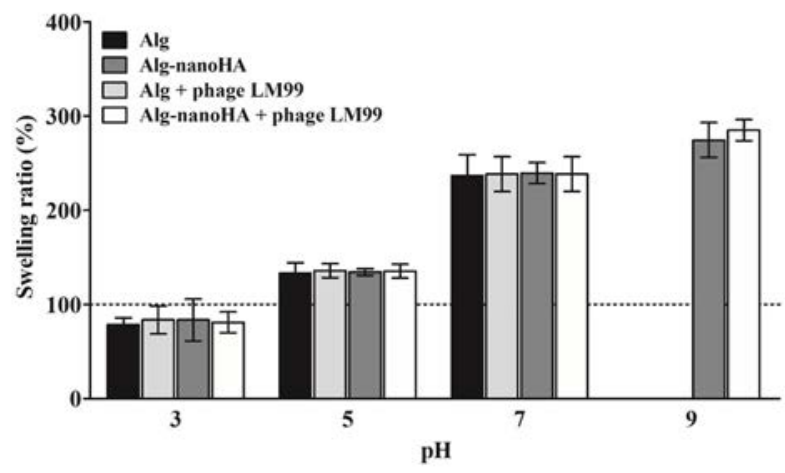

A2)

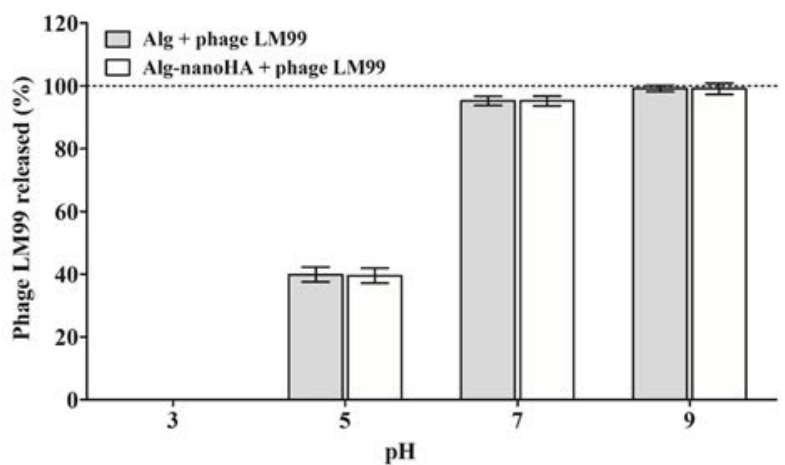

B)

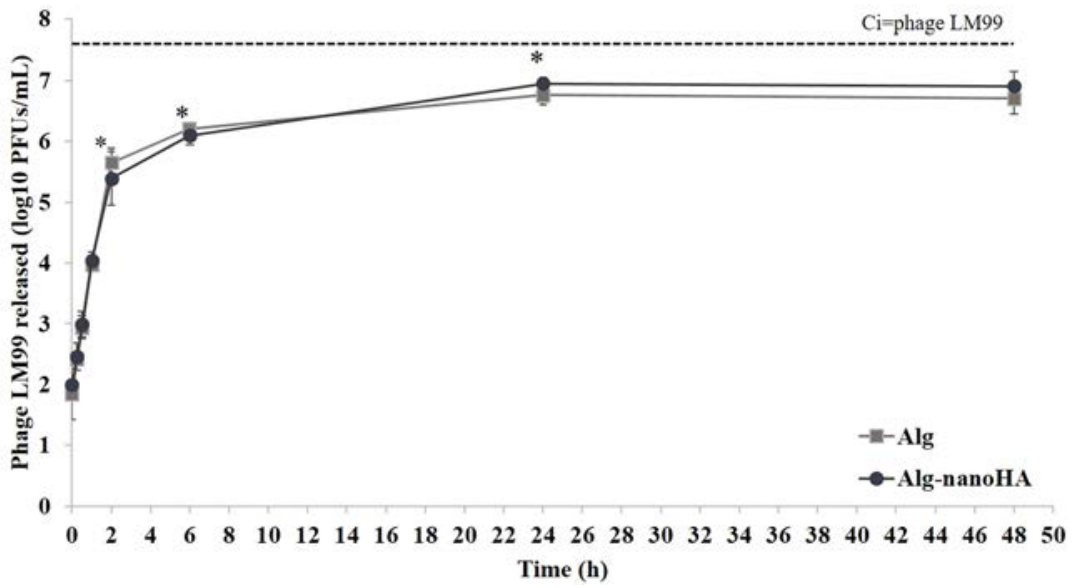

Figure 3. (A) Effect of $\mathrm{pH}$ on swelling behavior and associated phages released from hydrogel system. (B) Kinetics of phages released from the hydrogel system. Quantitative data are expressed in mean \pm SD. *Significant differences between the amount of phages released over time, for same sample (Alg or Alg-nanoHA) $(P<0.05)$.

media was changed daily. At the end of the experimental period, femurs were washed twice in PBS and fixed in 4\% paraformaldehyde. Histological analyses were performed following Alcian blue/Sirius red (AB/SR), von Kossa and Masson's trichrome staining. Images were captured with an Olympus BX-51/22 dotSlide digital virtual microscope. Phages-free Alg and AlgnanoHA hydrogels were used as controls. Three independent experiments were performed in quadruplicates.

\section{Antimicrobial activity}

In vitro characterization over planktonic and sessile bacteria

Antimicrobial activity of the hydrogel system was studied against pathogenic multidrug-resistant $E$. faecalis 201, obtained from orthopedic implant-related infections. ${ }^{24}$ Exponential bacterial cultures $\left(10^{7} \mathrm{CFU} / \mathrm{mL}\right)$ were incubated with the hydrogels at $37^{\circ} \mathrm{C}, 150 \mathrm{rpm}$ for $24 \mathrm{~h}$. After incubation, the planktonic and sessile population (on hydrogels and on tissue culture plates (TCPs)) were quantified by the colony-forming units' (CFUs) method. Bacterial growth percentage was plotted against time. Phages-free Alg and Alg-nanoHA hydrogels were used as controls. Three independent experiments were performed in triplicate.
Ex vivo antimicrobial assay in bacteria-infected femoral model

The antimicrobial activity was also evaluated through ex vivo chicken femoral model. Embryonic femurs with established defect margins were contaminated with multidrug-resistant $E$. faecalis $201(106 \mathrm{cell} / \mathrm{mL})$, for $10 \mathrm{~min}$, at room temperature. Then, phages-loaded hydrogels were implanted in the contaminated femurs and were carefully placed into NetwellTM Insert in six-well-plates. After $48 \mathrm{~h}$ of incubation, the bacterial colonization was characterized by histological analysis following Gram staining. Additionally, at 24 and $48 \mathrm{~h}$, the planktonic populations were quantified by CFUs method, plotting bacteria growth percentages against time. Phages-free Alg and AlgnanoHA hydrogels were used as controls. Three independent experiments were performed in quadruplicate.

\section{Statistical analysis}

Experimental data were analyzed using IBM ${ }^{\circledR}$ SPSS ${ }^{\circledR}$ Statistics (vs.22.0, SPSS, USA). Results were reported as the mean \pm standard deviation. One-way analysis of variance (ANOVA) followed by post hoc Tukey HSD multiple comparison tests were used to determine significant differences $(P<0.05)$. 



Figure 4. Histological images of subcutaneous tissue with implanted hydrogels stained with hematoxylin (the cell nucleic in blue) and eosin (extracellular matrix and cytoplasm in pink), at 2 and 6 weeks of implantation. Scale bars $100 \mu \mathrm{m}$.

\section{Results}

\section{Hydrogel system physico-chemical characterization}

Figure 1 shows the hydrogel system structure and network organization. Regardless of the encapsulated phages, Alg hydrogels showed a smooth surface, whereas Alg-nanoHA showed a rougher one. NanoHA were well embedded and homogeneously dispersed within the polymeric matrix (Figure 1A). All samples showed a typical Alg matrix, a network of fibril-like structures, (Figure 1B). The phages LM99 were entrapped within the Alg matrix (Figure 1B), for both Alg and Alg-nanoHA hydrogels. The integrity of phages' structure (icosahedral head and long-tail) was maintained after encapsulation, as observed in Figure 1C.

The ATR-FTIR spectra (Figure 2A) show the hydrogel system chemical composition. Regardless of phages encapsulation, Alg hydrogel displayed carboxylic group $\left(\mathrm{COO}^{-}\right)$at 1592 and $1417 \mathrm{~cm}^{-1}$, and hydroxyl group $\left(\mathrm{OH}^{-}\right)$between 3311 to 3307 $\mathrm{cm}^{-1}$. The bending of the $\mathrm{OH}^{-}$group of the carboxyl is depicted at $819 \mathrm{~cm}^{-1}$. ATR-FTIR spectra for phage-free and phage-loaded Alg-nanoHA hydrogels were also similar. These presented the peaks corresponding to the $\mathrm{Alg}$ matrix $\left(\mathrm{COO}^{-}\right.$bands), at the same wavelengths described for Alg hydrogels (Figure 2A). Peaks corresponding to phosphate group $\left(\mathrm{PO}_{4}^{3-}\right)$ were observed at $1020 \mathrm{~cm}^{-1}$ and $559 \mathrm{~cm}^{-1}$, attributed to the overlapping of $\mathrm{COO}^{-}$ stretching of $\mathrm{Alg}$ and $\mathrm{PO}_{4}^{3-}$ stretching of nanoHA. The presence of bands at $3365 \mathrm{~cm}^{-1}$ and $629 \mathrm{~cm}^{-1}$ were assigned to $\mathrm{OH}^{-}$ group, corresponding to lattice water (Figure 2A).

\section{Functional characterization}

Phages encapsulation efficiency and hydrogel system shelf-life assessment

High amount of phages LM99 was encapsulated in Alg and Alg-nanoHA hydrogels, with around $8.1 \log _{10} \mathrm{PFU} / \mathrm{mL}$, supporting a mean phage encapsulation efficiency of 91\% (Figure 2B).
The encapsulation did not cause detrimental effects in phages' activity and replication ability.

The shelf-life of encapsulated phages LM99 in solutions (Figure 2C), and in hydrogels (Figure 2D) was studied over time. In solution, phages titer remained constant $\left(8.3 \log _{10} \mathrm{PFU} / \mathrm{mL}\right)$ over 60 days, without alteration in phages' stability and viability between Alg and Alg-nanoHA solutions (Figure 2C). Regarding hydrogels, the amount of viable phages was also found to be broadly constant $\left(6.9 \log _{10} \mathrm{PFU} / \mathrm{mL}\right)$ during 7 -days, without differences between Alg and Alg-nanoHA (Figure. 2D).

Effect of $\mathrm{pH}$ on swelling behavior and associated phages release from the hydrogel system

The effect of different $\mathrm{pH}$ values in hydrogels' swelling behavior (Figure 3A1) and consequent phage release (Figure 3A2) is shown in Figure 3A. Regardless encapsulated phages, under acidic conditions, the protonation of the $\mathrm{COO}^{-}$groups of the Alg network occurs, promoting hydrogels shrinkage. Under alkaline conditions occurs the ionization of the $\mathrm{COO}^{-}$groups, endorsing the swelling of the hydrogels (Figure 3A1). A correlation between the swelling ratio (Figure 3A1) and released phages (Figure 3A2), was established (Figure 3). At pH3, shrinkage of $\mathrm{Alg}$ and Alg-nanoHA hydrogels matrix was observed (Figure 3A1), and no phages were detected (Figure $3 \mathrm{~A} 2$ ). Increasing $\mathrm{pH}$, progressive swelling behavior was observed, with disintegration occurring at alkaline $\mathrm{pH}$ values (Figure 3A1). At pH5, the swelling ratio was $130 \%$ (Figure $3 \mathrm{~A} 1$ ), allowing the release of $40 \%$ of the encapsulated phages in both hydrogels (Figure 3A2). At $\mathrm{pH} 7$, the swelling ratio was $230 \%$ and $95 \%$ of encapsulated phages were released from both hydrogels (Figure 3A). Finally, at pH9, breakdown of the Alg hydrogels was observed, while Alg-nanoHA30 continued to swell. The released phages titer from Alg-nanoHA at $\mathrm{pH} 9$ was 95\% (Figure 3A2). Overall, LM99 phages showed higher stability in the $\mathrm{pH}$ range $5-9$, with a $100 \%$ survival rate, while, at $\mathrm{pH} 3$ phages were completely inactivated. 
A)
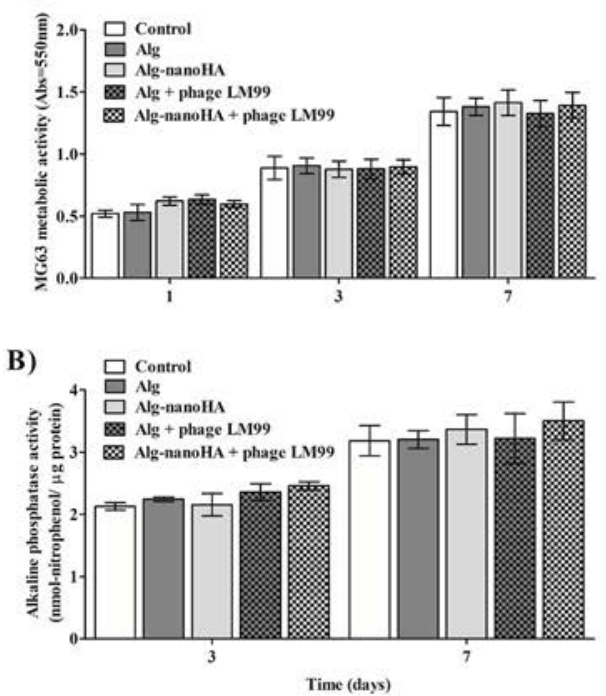

C)
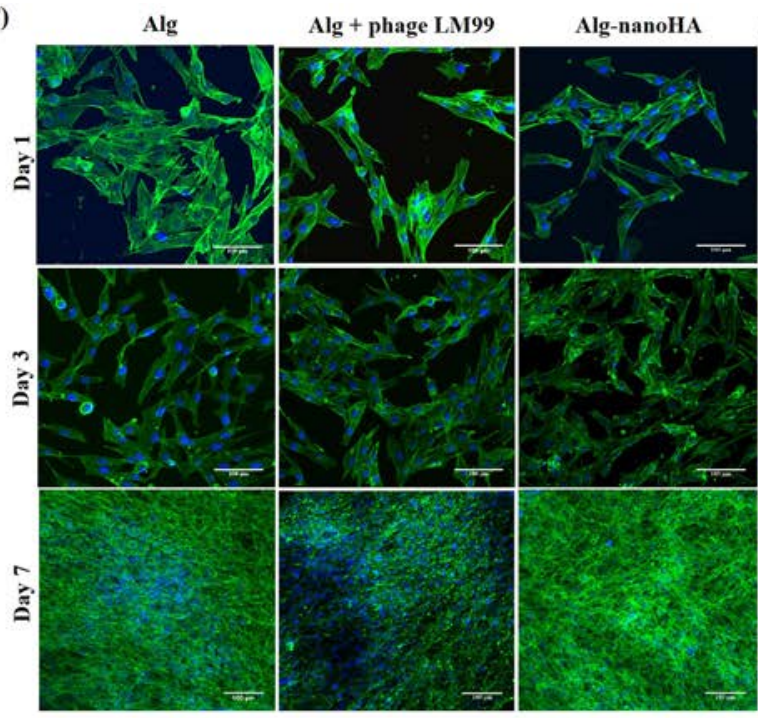

Alg-nanoHA + phage LM99

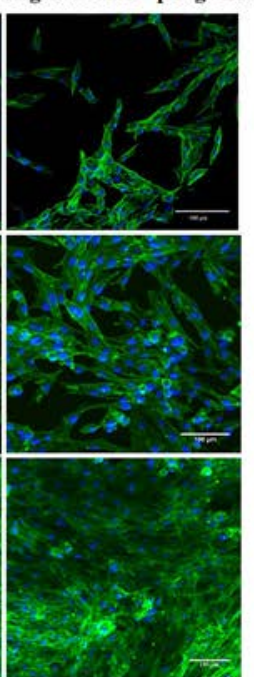

Figure 5. (A) Cell metabolic activity and (B) ALP activity of osteoblastic cells exposed to hydrogels eluents. Quantitative data are expressed in mean \pm SD. (C) Immunofluorescent images of osteoblastic cells exposed to hydrogels' eluents. The cytoskeleton was stained green and the nucleus counterstained in blue. Scale bar $100 \mu \mathrm{m}$.

\section{Profile of phages LM99 release}

In Figure 3B the profile of phages LM99 release from hydrogels is shown. No differences in released phages titer between Alg and Alg-nanoHA hydrogels were observed over time. A gradual increase in the phage titer was observed for $24 \mathrm{~h}$. After $30 \mathrm{~min}, 40 \%$ of encapsulated phages were released and after 6 and $24 \mathrm{~h}$ of incubation, $88 \%$ and $97 \%$ of phages were released, respectively. After this time point, no differences were observed in the amount of phages, with almost all phages being released from Alg and Alg-nanoHA hydrogels (Figure 3B).

\section{Biological characterization}

Inflammatory response - in vivo subcutaneous tissue implantation

Throughout the follow-up period, no post-operative complications as tissue infection or other adverse host reactions were identified (Figure 4). At the defined time points, animals were euthanized and a systematic necropsy was carried out, revealing no macroscopic alterations of internal organs. Also, at the implantation site, no significant inflammatory reaction or cellular exudate was identified.

After 2 weeks, tissue surrounding implanted hydrogels, phages-free and phages-loaded, displayed symptoms of a mild immune activation. Moreover, all implanted hydrogels were surrounded by a capsular organized structure, rich in fibroblasts and collagen fibers. The cells population within the hydrogels at two weeks consisted mainly of polymorphonuclear leukocytes, lymphocytes, and macrophages, with no evidence of bleeding, intercellular edema, or vascular congestion (Figure 4). Comparatively, no differences in the immune response, recruited cell populations, and tissue infiltration were observed between all conditions at six weeks, while an increased hydrogel fragmentation was observed, with tissue infiltration established throughout the fragments of the implanted hydrogels. Again, no phage encapsulation was found to modify the obtained cell response on Alg and Alg-nanoHA hydrogels (Figure 4).

\section{Osteogenic response}

In vitro cytocompatibility assessment with human osteoblastic cells

An increase in MTT reduction values was observed in the control, throughout the 7-days culture period. Comparatively, no significant differences were found between cells exposed to hydrogels eluents, phages-free or phages-loaded, and no differences were found between them and the reference control (free-hydrogel eluents) (Figure 5A).

Functional activity of grown osteoblastic cultures, determined by ALP activity, was found to increase between days 3 and 7 of culture, with highest levels being obtained at day 7 , in cultures grown on control (Figure 5B). Cultures grown in the presence of hydrogels eluents, phages-free or phages-loaded, presented similar results, and no significant differences were found between the several conditions (Figure 5B).

Cells massively adhered to culture substrates, presenting an elongated and spread morphology with evident cell-to-cell contact being established since day 1 (Figure 5C). Cells further exhibited well-organized $\mathrm{F}$-actin cytoskeletons and prominent central nuclei, with philopodia extending at the cell margins. Representative images at day 3 revealed an active proliferation, which further increased until day 7 , when organized cell layers could be depicted, with highly confluent zones. The cells exposed to hydrogels eluents, phages-free or phages-loaded, showed similar morphological organization within the reference cultures.

\section{Ex vivo functional assessment of bone formation}

Phages-free and phages-loaded Alg hydrogels induced a moderate osteogenic activation at the margin of the established segmental defect, and also at the bone collar formed within the 

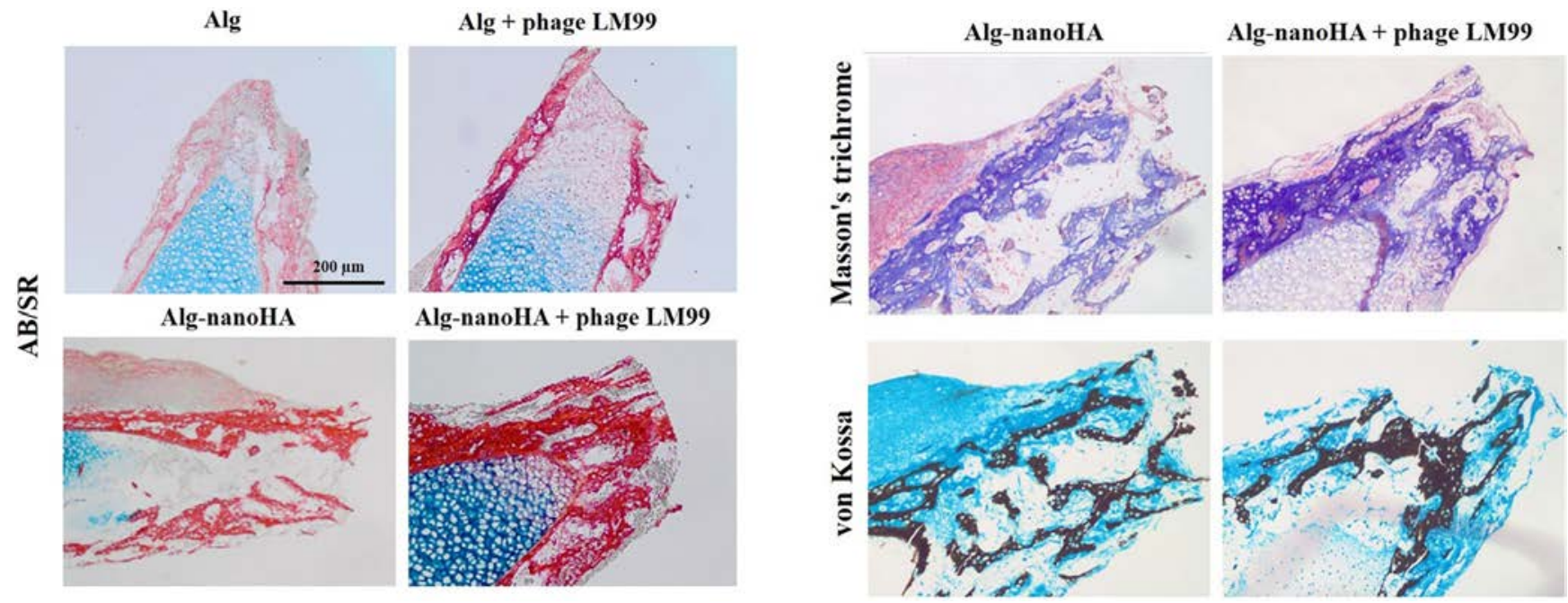

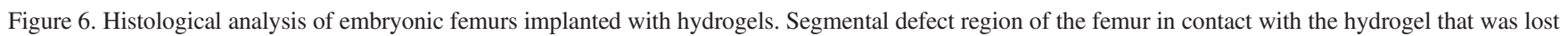

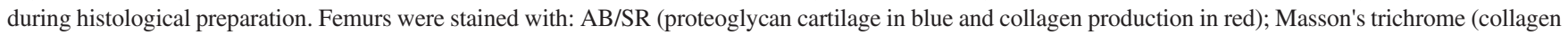
fibers in blue), and van Kossa (bone mineralization in black). Scale bar at $200 \mu \mathrm{m}$.

tissue peripheral structure (Figure 6). Comparatively, an increased osteogenic response was observed for Alg-nanoHA hydrogels, regardless the presence of phages. The implantation of Alg-nanoHA enhanced significantly collagenous matrix deposition within the central and marginal regions of the diaphysial structure and an organized and thick trabecular structure was formed. Masson's trichrome staining further corroborated increased collagenous deposition observed with Alg-nanoHA hydrogels. Increased mineral deposition within the developed trabecular structure was also evidenced by the von Kossa staining. It should be noticed that phage encapsulation seems not to have interfered with the enhanced osteogenic capability of Alg-nanoHA hydrogels.

\section{Antimicrobial activity}

In vitro characterization over planktonic and sessile bacteria

The antimicrobial activity of the hydrogel system against planktonic and sessile bacteria is depicted in Figure 7.

In the presence of phages-free hydrogels, an increase in planktonic and sessile population was observed after $24 \mathrm{~h}$, and no significant differences between $\mathrm{Alg}$ and Alg-nanoHA hydrogels could be found.

In the presence phages-loaded hydrogels, planktonic bacteria were significantly inhibited (around 99\%), after 24h incubation (Figure 7A). Regarding sessile bacteria into hydrogels and in TCPs, the phages-loaded hydrogels showed the same antimicrobial behavior. Phages-loaded hydrogels were further able to reduce the bacterial attachment in $98 \%$ on hydrogels and $92 \%$ on TCPs. No differences in antimicrobial activity were found between Alg + LM99 and Alg-nanoHA + phage LM99 hydrogels.

Ex vivo antimicrobial assay in bacteria-infected femoral model

Antibacterial activity was also evaluated using an ex vivo chicken femoral model, previously infected with multi-resistant E. faecalis 201 strain (Figure 8).
In the presence phages-loaded hydrogels, bacterial density was 3-orders of magnitude lower in terms of CFUs when compared to phage-free hydrogels, corresponding to inhibitions of $99.6 \%$ and $99.9 \%$ after 24 and 48 h, respectively (Figure $8 \mathrm{~A}$ ). No differences in antimicrobial activity were found between Alg + LM99 and Alg-nanoHA + phage LM99 hydrogels.

Bacterial colonization within femurs was dependent on the hydrogels implanted within a segmental defect (Figure 8B). Regarding phages-free hydrogels implantation, bacterial colonies were identified within the femurs tissue structure (Figure 8B - a.2, a.2.1, a.2.2, b.2, b.2.1 and b.2.2), as revealed by Gram staining. Contrariwise, no bacterial growth was noticed when phages-loaded hydrogels were implanted (Figure $8 \mathrm{~B}$ - c.2, c.2.1, c.2.2, d.2, d.2.1 and d.2.2).

\section{Discussion}

Any surgical intervention, particularly including biomaterials implantation, is associated to high risk of infection, which can be devastating for the patients, generating overload healthcare systems. $^{29}$ Antimicrobial-loaded biomaterials, as delivery systems, have received special attention in applications for prevention and treatment of implant-related infections. ${ }^{9}, 29$ However, limited antimicrobial diffusion, sub-therapeutic levels of antimicrobial agent, dose-dependent antimicrobial activity, antimicrobials' toxicity, and the spread of antibiotic-resistant bacteria, underscore the need for new approaches to control these bacterial infections. ${ }^{6,7,9,30}$

In this study, a new drug-delivery system based on encapsulated phages on Alg-nanoHA hydrogels was assessed as a multifunctional approach. This system has three components with specific and complementary functionalities: 1) Alg hydrogel is responsible for transporting and delivering the therapeutic agents to the local target, 2) osteo-regeneration stimulation is endorsed by the presence of bioactive and osteoconductive nanoHA, and, 3) 


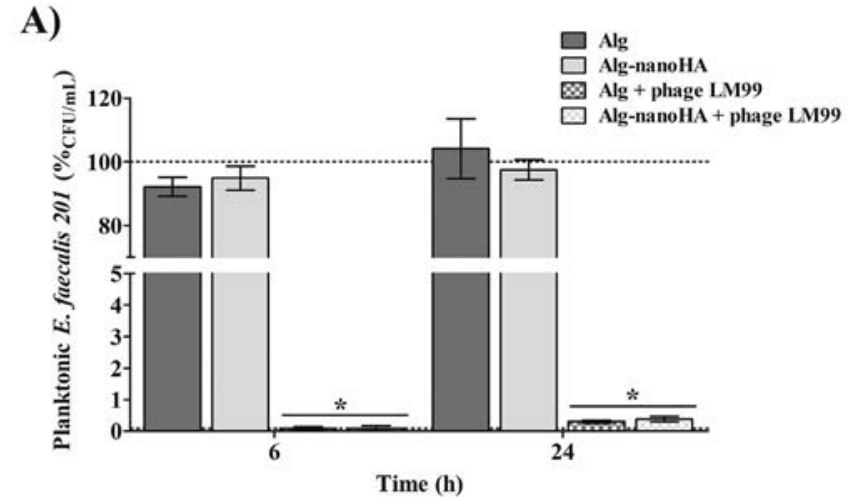

B)

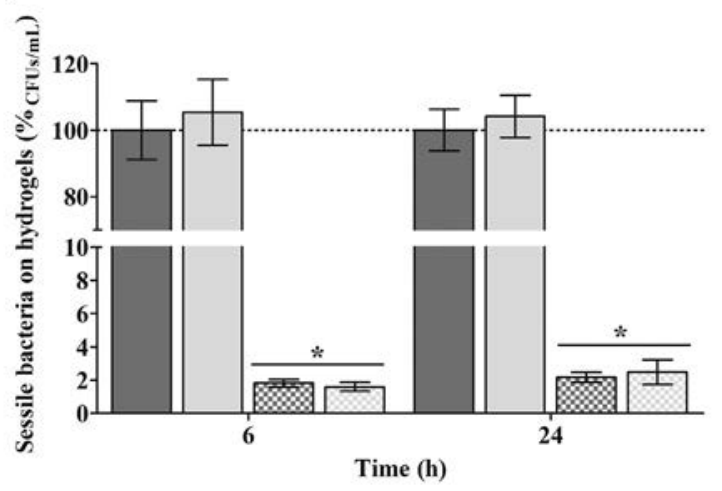

C)

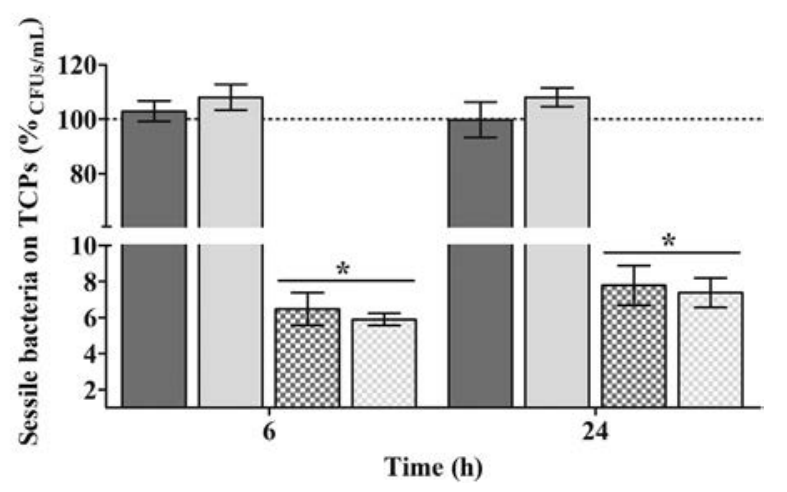

Figure 7. In vitro antimicrobial activity: (A) Planktonic E. faecalis 201 growth in the presence of hydrogels; (B) Sessile bacteria on hydrogels, and (C) Sessile bacteria on TCPs. Quantitative data are expressed in mean \pm SD. *Significant differences of bacterial density between phages-free and phages-loaded hydrogels, for same sample (Alg or Alg-nanoHA) at 6 and $24 \mathrm{~h}$, respectively $(P<0.05)$.

prevention of bacterial colonization and proliferation is addressed by the local delivery of phages.

In the present work, it was shown that phages were entrapped into the three-dimensional hydrogel network, by ionic crosslinking, without jeopardizing phage morphology, viability and functionality. A remarkable shelf-life of encapsulated phages on Alg or Al-nanoHA solutions was observed, whereas the shelflife of phages into hydrogels was also broadly unaltered up to 7 days. Other authors also reported the encapsulation of phages FelixO1 and phages UAB_Phi20 into Alg hydrogels with similar behavior and efficiency. ${ }^{26,31}$ The Alg solubility capacity could explain the phage delivering from the hydrogel system. When, drug-loaded Alg hydrogels are exposed to an aqueous medium, a successive progression of swelling-disintegration-erosion occurs at the Alg network by ion-exchange with the medium, allowing the drug to diffuse out of the hydrogel. ${ }^{32}$ Several authors have reported the importance of $\mathrm{pH}$-dependence and hydrophilic behavior of Alg network, on drug release mechanisms. ${ }^{31,33,34}$ For instance, at basic $\mathrm{pH}$, the swelling of Alg network occurs, due to the ionization of the $\mathrm{COO}^{-}$groups and diffusion of $\mathrm{Ca}^{2+}$ ions from gelling sites, ${ }^{31}, 33,34$ allowing thereby the phages permeability. Whereas, under acid conditions the Alg network shrinks, due to the protonation of the $\mathrm{COO}^{-}$groups and increase of cross-linking degree, ${ }^{31,33,34}$ holding the phages entrapped in Alg matrices. It should be noted that no detrimental effects on phages viability and functionally were observed after phages delivery at broad range of $\mathrm{pH} 5-9$, which could be a promising feature for phages delivery at the site of bone infection, where the normal $\mathrm{pH}$ is $6.8 .^{35}$ Besides, it was shown that the presence of nanoHA into the hydrogel system did not affect the phage releasing from hydrogels, with almost all phages being released from the composites, suggesting Alg-nanoHA hydrogels could be used as a suitable phages release delivery system. ${ }^{31,33,34}$ Similar phages release profiles were reported from hydroxypropylmethylcellulose, alginate/ $\mathrm{CaCO}_{3}$ or Eudragit ${ }^{\circledR}$ hydrogels for targeted delivery of phages. ${ }^{9}, 23,31,34$

Biocompatibility and safety are important requirements for local drug delivery systems and their tissue engineering applications. ${ }^{36}$ In the present work, good biocompatibility and safety profiles were observed for all hydrogels subcutaneously implanted in a rabbit model. Some reports have shown the efficiency of phages therapy in medical, veterinary, agricultural, and aquaculture applications without adverse safety concerns for eukaryotic cells. $^{13,37,38}$ Phages administration has shown to stimulate the host immune response, depending on the route of administration, e.g., oral and topical administrations may induce the production of anti-phages neutralizing antibodies, while intravenous administration of phages has the potential to stimulate innate and adaptive immune responses. ${ }^{13}, 39$ No significant differences were identified on the local tissue response following the implantation of phages-loaded hydrogels, sustaining their biosafety profile. 

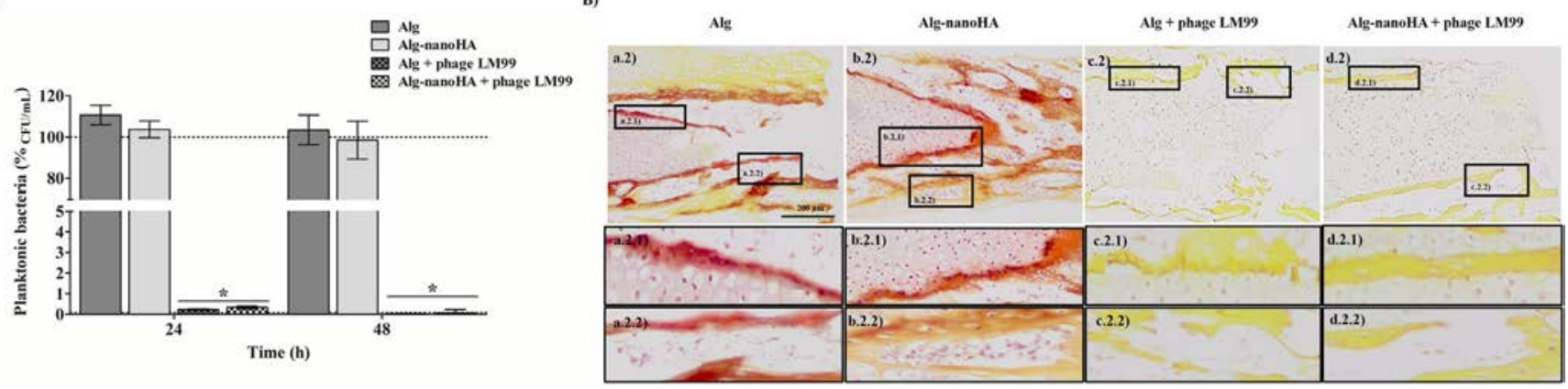

Figure 8. Ex vivo antimicrobial activity: (A) E. faecalis 201 density growth surrounding femurs implanted with hydrogels. Quantitative data are expressed in mean \pm SD. *Significant differences in bacterial proliferation between femurs implanted with phages-free and phages-loaded hydrogels, for same sample (Alg or Alg-nanoHA) at 24 and $48 \mathrm{~h}$, respectively $(P<0.05)$. (B) Histological analysis of bacterial colonization within the implanted femurs, following Gram staining. Bacterial clusters were observed in the trabeculae (a.2.1 and b.2.1) and in the underlying spaces (a.2.2 and b.2.2) of the femoral tissues implanted with phagefree-hydrogels (a.2 and b.2). Scale bar at $200 \mu \mathrm{m}$.

Moreover, by in vitro cytocompatibility, it was observed that phages and hydrogels' leachable products did not interfere with osteoblastic cells' viability, proliferation and morphology, indicating that the phage-loaded biomaterial systems could allow for bone cells spreading and growth. Chung et al suggested that incorporation of phages into tissue engineering fibers did not interfere with cell behavior. ${ }^{40}$ The cytocompatibility of Alg and nanoHA materials was previously described by other studies. ${ }^{33,41}$

The osteogenic response of hydrogel systems was emphasized by ex vivo functionality of bone formation results. Regardless the presence of phages, the Alg-nanoHA hydrogels implantation significantly increased the osteogenic and mineralization response, highlighting the potential of nanoHA as an osteoconductive and osteoinductive agent. Other studies have shown that nanoHA is able to enhance bone ingrowth and accelerated bone formation within and around the implanted material. ${ }^{42,}{ }^{43}$ The ex vivo data suggest that Alg-nanoHA + phage LM99 hydrogels could be used to improve bone tissue regeneration at local bone defects.

To the best of our knowledge, the present work is the first to develop a drug-delivery system based on encapsulated phages on Alg-nanoHA hydrogel to prevent and control bacterial contamination during bone implantation and integration. In the present work, the antimicrobial activity of phage LM99 encapsulated on Alg-nanoHA hydrogels was evaluated against multidrugresistant E. faecalis 201 bacteria, under both in vitro and ex vivo conditions, as proof of concept. Under in vitro conditions, effective antimicrobial activity and large diffusion of phages LM99 from hydrogels were observed, inhibiting attachment and growth of planktonic and sessile E. faecalis 201 on biomaterials and surrounding media. Besides, in ex vivo assessment, it was shown that phage LM99-loaded hydrogels prevented proliferation and colonization of the multidrug-resistant E. faecalis 201 in the neighborhood of the implant and within the femoral tissues. Barros et al described the effectiveness of phage therapy in the prophylaxis and treatment of vancomycin-resistant $E$. faecalis, methicillin-resistant $S$. aureus (MRSA) and E. coli isolated from orthopedic implant-related infections. ${ }^{24}$ Additionally, in the present study, lytic phages $\mathrm{LM} 12^{44}$ and $\mathrm{JB} 75^{24}$ were also encapsulated in the developed hydrogels, and their antimicrobial activity was evaluated in vitro against multidrug-resistant bacteria involved in implant-related infections ${ }^{24}$ (see Supplementary material). The results showed that the release of infecting phages LM12 and JB75, to the planktonic phase, was able to kill suspended MRSA 80 and E. coli 75, respectively (Supplementary material). The obtained results further emphasize the therapeutic potential of the developed local delivery approach, in the prevention and eradication of relevant bacteria strains, even those showing multi-drug resistance profiles, at the implant microenvironment, prior to their adhesion and biofilm establishment.

Overall, a new multifunctional drug-delivery system based on encapsulated phages on Alg-nanoHA hydrogels was developed and showed to be a promising and efficient approach to prevent and control bacterial contamination during implantation and bone integration.

\section{Authors' Contribution}

JB drafted the main manuscript and performed experiments and data analysis. LDRM participated in the data analysis and helped in manuscript writing. RAS, VP, BC, MHF and PSG took part in the design of the study, helped in the in vivo and ex vivo assays performance and analysis, and help in manuscript writing. MPF, JA and FJM participated in the data analysis and critically revised the manuscript. All authors read and approved the manuscript.

\section{Appendix A. Supplementary data}

Supplementary data to this article can be found online at https://doi.org/10.1016/j.nano.2019.102145.

\section{References}

1. Wang W, Yeung KWK. Bone grafts and biomaterials substitutes for bone defect repair: A review. Bioact Mater 2017;2(4):224-47. 
2. Zimmerli W, Moser C. Pathogenesis and treatment concepts of orthopaedic biofilm infections. FEMS Immunol Med Microbiol 2012;65(2):158-68.

3. Gimeno M, Pinczowski P, Perez M, Giorello A, Martinez MA, Santamaria J, et al. A controlled antibiotic release system to prevent orthopedic-implant associated infections: An in vitro study. Eur $J$ Pharm Biopharm 2015;96:264-71.

4. Street TL, Sanderson ND, Atkins BL, Brent AJ, Cole K, Foster D, et al. others. Molecular diagnosis of orthopedic-device-related infection directly from sonication fluid by metagenomic sequencing. $J$ Clin Microbiol 2017;55(8):2334-47.

5. Junka A, Szymczyk P, Ziolkowski G, Karuga-Kuzniewska E, Smutnicka $\mathrm{D}$, Bil-Lula I, et al. Bad to the bone: on in vitro and ex vivo microbial biofilm ability to directly destroy colonized bone surfaces without participation of host immunity or osteoclastogenesis. PLoS One 2017;12 (1)e0169565.

6. Qiu Y, Zhang N, An YH, Wen X. Biomaterial strategies to reduce implant-associated infections. Int $J$ Artif Organs 2007;30 (9):828-41

7. Silva T, Silva JC, Colaco B, Gama A, Duarte-Araujo M, Fernandes MH, et al. In vivo tissue response and antibacterial efficacy of minocycline delivery system based on polymethylmethacrylate bone cement. $J$ Biomater Appl 2018;33(3):380-91.

8. Francolini I, Vuotto C, Piozzi A, Donelli G. Antifouling and antimicrobial biomaterials: an overview. APMIS 2017;125(4):392-417.

9. Kaur S, Harjai K, Chhibber S. Bacteriophage mediated killing of Staphylococcus aureus in vitro on orthopaedic $\mathrm{K}$ wires in presence of linezolid prevents implant colonization. PLoS One 2014;9(3).

10. Loc-Carrillo C, Abedon ST. Pros and cons of phage therapy. Bacteriophage 2011;1(2):111-4.

11. Edgar R, Friedman N, Molshanski-Mor S, Qimron U. Reversing bacterial resistance to antibiotics by phage-mediated delivery of dominant sensitive genes. Appl Environ Microbiol 2012;78(3):744-51.

12. Nobrega FL, Costa AR, Kluskens LD, Azeredo J. Revisiting phage therapy: new applications for old resources. Trends Microbiol 2015;23 (4):185-91.

13. Morris J, Kelly N, Elliott L, Grant A, Wilkinson M, Hazratwala K, et al. Evaluation of bacteriophage anti-biofilm activity for potential control of orthopedic implant-related infections caused by Staphylococcus Aureus. Surg Infect 2019;20(1):16-24.

14. Yilmaz C, Colak M, Yilmaz BC, Ersoz G, Kutateladze M, Gozlugol M. Bacteriophage therapy in implant-related infections an experimental study. J Bone Jt Surg 2013;95a(2):117-25.

15. Trampuz A, Klatt A, Luca MD. Isolation of new lytic bacteriophages fro treatment of prosthetic joint infections. J Bone Joint Surg 2017;99-B (SUPP_22):36-36.

16. Oliveira H, Sillankorva S, Merabishvili M, Kluskens LD, Azeredo J. Unexploited opportunities for phage therapy. Front Pharmacol 2015;6:180.

17. Gorski A, Jonczyk-Matysiak E, Miedzybrodzki R, Weber-Dabrowska B, Lusiak-Szelachowska M, Baginska N, et al. Phage therapy: beyond antibacterial action. Front Med (Lausanne) 2018;5:146.

18. Fish R, Kutter E, Bryan D, Wheat G, Kuhl S. Resolving digital staphylococcal osteomyelitis using bacteriophage - a case report. Antibiotics (Basel) 2018;7(4).

19. Kishor C, Mishra RR, Saraf SK, Kumar M, Srivastav AK, Nath G. Phage therapy of staphylococcal chronic osteomyelitis in experimental animal model. Indian J Med Res 2016;143(1):87-94.

20. Dublanchet A. Patey O. Phage therapy for bone and joint infections: report of french cases. Orthopaedic Proceedings 2017;99-B (SUPP_22):35-35.

21. Chan BK, Turner PE, Kim S, Mojibian HR, Elefteriades JA, Narayan D. Phage treatment of an aortic graft infected with Pseudomonas aeruginosa. Evol Med Public Health 2018;1:60-6.

22. Meurice E, Rguiti E, Brutel A, Hornez JC, Leriche A, Descamps M, et al. New antibacterial microporous $\mathrm{CaP}$ materials loaded with phages for prophylactic treatment in bone surgery. J Mater Sci Mater Med 2012;23 (10):2445-52.

23. Kaur S, Harjai K, Chhibber S. In vivo assessment of phage and linezolid based implant coatings for treatment of methicillin resistant $S$. aureus (MRSA) mediated orthopaedic device related infections. PLoS One 2016;11(6).

24. Barros J, Melo LDR, Poeta P, Igrejas G, Ferraz MR, Azeredo J, et al. Lytic bacteriophages against multidrug-resistant Staphylococcus aureus, Enterococcus faecalis and Escherichia coli isolates from orthopaedic implant-associated infections. Int J Antimicrob Agents 2019;54 (3):329-37.

25. Melo LDR, Sillankorva S, Ackermann HW, Kropinski AM, Azeredo J, Cerca N. Characterization of Staphylococcus epidermidis phage vB_SepS_SEP9-a unique member of the Siphoviridae family. Res Microbiol 2014;165(8):679-85.

26. Ma Y, Pacan JC, Wang Q, Xu Y, Huang X, Korenevsky A, et al. Microencapsulation of bacteriophage felix O1 into chitosan-alginate microspheres for oral delivery. Appl Environ Microbiol 2008;74 (15):4799-805.

27. Mohammed NGrishkewich, Berry R, Tam K. Cellulose nanocrystalalginate hydrogel beads as novel adsorbents for organic dyes in aqueous solutions. Cellulose 2015;22(6):3725-38.

28. Gomes PS, Fernandes MH. Effect of therapeutic levels of doxycycline and minocycline in the proliferation and differentiation of human bone marrow osteoblastic cells. Arch Oral Biol 2007;52 (3):251-9.

29. Orapiriyakul W, Young PS, Damiati L, Tsimbouri PM. Antibacterial surface modification of titanium implants in orthopaedics. J Tissue Eng 2018;9.

30. Siljander MP, Sobh AH, Baker KC, Baker EA, Kaplan LM. Multidrug-resistant organisms in the setting of periprosthetic joint infectiond-diagnosis, prevention, and treatment. J Arthroplast 2018;33(1):185-94.

31. Colom J, Cano-Sarabia M, Otero J, Arinez-Soriano J, Cortes P, Maspoch D, et al. Microencapsulation with alginate/ $\mathrm{CaCO}_{3}$ : A strategy for improved phage therapy. Sci Rep 2017;741441.

32. Tønnesen HH, Karlsen J. Alginate in drug delivery systems. Drug Dev Ind Pharm 2002;28(6):621-30.

33. Jain D, Bar-Shalom D. Alginate drug delivery systems: application in context of pharmaceutical and biomedical research. Drug Dev Ind Pharm 2014;40(12):1576-84.

34. Vinner GK, Vladisavljevic GT, Clokie MRJ, Malik DJ. Microencapsulation of Clostridium difficile specific bacteriophages using microfluidic glass capillary devices for colon delivery using $\mathrm{pH}$ triggered release. PLoS One 2017;12(10).

35. Kinnari TJ, Esteban J, Martin-De-Hijas NZ, Sanchez-Munoz O, Sanchez-Salcedo S, Colilla M, et al. Influence of surface porosity and $\mathrm{pH}$ on bacterial adherence to hydroxyapatite and biphasic calcium phosphate bloceramics. J Med Microbiol 2009;58(1):132-7.

36. ter Boo GJA, Grijpma DW, Moriarty TE, Richards RG, Eglin D. Antimicrobial delivery systems for local infection prophylaxis in orthopedic- and trauma surgery. Biomaterials 2015;52:113-25.

37. Cisek AA, Dabrowska I, Gregorczyk KP, Wyzewski Z. Phage therapy in bacterial infections treatment: one hundred years after the discovery of bacteriophages. Curr Microbiol 2017;74(2):277-83.

38. Kazmierczak Z, Gorski A, Dabrowska K. Facing antibiotic resistance: Staphylococcus aureus phages as a medical tool. Viruses-Basel 2015;7(4):1667.

39. Zimecki M, Artym J, Kocieba M, Weber-Dabrowska B, Borysowski J, Gorski A. Effects of prophylactic administration of bacteriophages to immunosuppressed mice infected with Staphylococcus aureus. BMC Microbiol 2009;9:169.

40. Chung W-J, Merzlyak A, Lee S-W. Fabrication of engineered M13 bacteriophages into liquid crystalline films and fibers for directional growth and encapsulation of fibroblasts. Soft Matter 2010;6 (18):4454-9. 
41. Teotia AK, Raina DB, Singh C, Sinha N, Isaksson H, Tagil M, et al. Nano-hydroxyapatite bone substitute functionalized with bone active molecules for enhanced cranial bone regeneration. ACS Appl Mater Interfaces 2017;9(8):6816-28.

42. Cunniffe GM, Curtin CM, Thompson EM, Dickson GR, O'Brien FJ. Content-dependent osteogenic response of nanohydroxyapatite: an in vitro and in vivo assessment within collagen-based scaffolds. ACS Appl Mater Interfaces 2016;8(36):23477-88.
43. Martinelli NM, Ribeiro MJG, Ricci R, Marques MA, Lobo AO, Marciano FR. In Vitro Osteogenesis stimulation via nano-hydroxyapatite/carbon nanotube thin films on biomedical stainless steel. Materials 2018;11(9).

44. Melo LDR, Brandao A, Akturk E, Santos SB, Azeredo J. Characterization of a new Staphylococcus aureus kayvirus harboring a lysin active against biofilms. Viruses-Basel 2018;10(4). 